\title{
A Model of Self-sustaining Magnetic Dynamo in Accretion Disks
}

\author{
Hongliang Zhao, Bo Jiang and Li Wang \\ Fundamental Department, Aviation University of Air Force, China \\ 294244476@qq.com
}

Keywords: Accretion; Accretion disks; Magnetic reconnection; Magnetic field; Magnetic dynamo

\begin{abstract}
This paper put forward a model of magnetic dynamo which similar to the model of C.A.Tout and J.E.Pringle, it explains the movement way of magnetic dynamo in accretion disks, and the main principle also are the instability of the Parker, the instability of the Balbus-Hawley and the reconnection. The difference is that the Petschek reconnection is substituted into Sweet-Parker reconnection, it can also form a self-sustaining magnetic dynamo.
\end{abstract}

\section{Introduction}

The initial stage of the theory of the dynamo has experienced twists and turns. One hand, the people always meet some difficulties in looking for the model of dynamo. On the other hand, some people build up many negative theorem, and suggest that the maintenance of the dynamo effect is impossible, being called the theorem of none dynamo. In 1934, Cowling suggested that the axisymmetric movement of the fluids couldn't maintain the meridian magnetic field, is called the theorem of Cowling. Latter, the people put up many theories being similar to the theorem of Cowling, the study of the theory of dynamo is in the hot water. In this condition, some people guess that the existence of the model of the self-sustaining dynamo is impossible. Practically, the theory of none dynamo puts forward many restrictive conditions that contribute to build up reasonable model of the dynamo. In 1955, Parker suggested that the differential rotation and the small-scale circumfluence in the star can maintain the progress of the self-sustaining dynamo. It breaks the stalemate of the study of the theory of the dynamo, and lays the physics foundation of the model of the dynamo of the turbulence. In 1964, some scholars researched the progress of the approximately axisymmetric model of the dynamo in order to explain the universal magnetic field. In 1966, Steenbeck, Krause and Radler develop a set of theory of the dynamo about the turbulence by putting forward the theory of electrodynamics which is an important development in the theory. For several decades, the theory of the dynamo about turbulence gets the booming development, because it not only can explain the maintaining of the magnetic field of the earth, but also can explain the period variety of the sun and the progress of the magnetic field in the universe. But the theory of the dynamo about turbulence is built up in the foundation of the first order in a smooth condition, in this condition, they ignore many coupling progress of the turbulence, because the people can't study the essential of the turbulence clearly, some people put forward many doubt about the dynamo about turbulence, and also put forward some new theorem.

Through several decades of development, the theory of dynamo as the explanation of the magnetic field in the universe is accepted widely. According to the ampere laws, the electricity can produce the magnetic field, since a majority of the material in the universe are ion, their movement correspond to electricity, also correspond to magnetic field. The suppositions that every according to this kind of way which assume that the movement of the electric material can produce magnetic field all belong to the theory of dynamo. At the early stage in 1919, Lamor suggested that the movement of the eddy around the sunpot could produce the magnetic field of sunpot. Although this theory is already abandoned, it educes many other theory of dynamo. The problem is that what form of the movement can produce stable magnetic field. In the past the people consider that the anomalous and disorderly turbulence can produce magnetic field, of course, this view can't make people convince. Think generally now, only the regular movement of the electrical material can produce magnetic field, but not all this kind of movement can produce magnetic field. For example, 
the movement of the axisymmetric electrical material can't maintain a stable magnetic field. In recent years, the theory of the dynamo avoid the mechanism of the theory of none dynamo and obtain an important development in the theory and application, but the biggest difficulty is that we have not yet study the essential of the turbulence clearly.

In 1966, Stenbeck and Radler put forward the electrodynamic theory and develop a set of theory of the dynamo about the turbulence. The magnetic field can be divided into the azimuthal field and radial field, so we must build up a physics mechanism that can produce the two weights of the magnetic field. Parker suggest that the tiny material of plasmas is continue to expand in the ascendable progress in the inner of the sun while it is revolving by the effect of coriolis force. If the tiny material take the flow tube of the magnetic to move upwardly, the twist of tiny material is to convert the azimuthal field $B_{\varphi}$ to the radial field $B_{r}$. The creation rate of $B_{r}$ is order to $B_{\varphi}$. Parker put the net effect of the convective tiny material to come down to add a electric field $E_{\varphi}$, namely $E_{\varphi}=\alpha B_{\varphi}$.

On this foundation, Krause and Radler discuss the statistic stability and the dynamo effect produced by the small-scale turbulence in 1980, and obtain the physics meaning of $\alpha$. They suggest that the $\alpha$ effect is the statistic property of the small-scale turbulence, in the condition that the flow speed is $v$ in the big scale and the dissipation speed $\eta$ is larger than the dissipation speed of the dynamics of the plasmas, the equation of the magnetic induction is:

$$
\frac{\partial \vec{B}_{0}}{\partial t}=\vec{\nabla} \times\left(\vec{v}_{0} \times \vec{B}_{0}\right)+\vec{\nabla} \times\left(\alpha \vec{B}_{0} \eta \vec{\nabla} \times \vec{B}_{0}\right)
$$

Obviously, for a axisymmetric flow, $v_{0}=\mathrm{R} \omega(\mathrm{R}, \omega) \varphi_{+} v_{r}$, when the equation of magnetic field write as $\mathrm{B}={ }^{B}{ }_{0}(\mathrm{R}, \omega) \varphi_{+} \nabla \times\left(A_{r}(\mathrm{R}, \omega) \varphi\right.$, it can use two scalar equations of the dynamo instead of the equation of magnetic induction:

$$
\begin{aligned}
& \frac{\partial \vec{B}_{\varphi}}{\partial t}+\mathrm{R}\left(\vec{v}_{r} \bullet \vec{\nabla}\right)\left[\frac{\vec{B}_{\varphi}}{R}\right]=\mathrm{R}\left(\vec{B}_{r} \bullet \vec{\nabla}\right) \omega_{+} \vec{\nabla} \times\left(\alpha \vec{B}_{r}\right)+\eta\left(\nabla^{2}-R^{-2}\right) \vec{B}_{\varphi} \\
& \frac{\partial \vec{A}_{r}}{\partial t}+\frac{1}{R}\left(\vec{v}_{r} \bullet \vec{\nabla}\right)\left(\mathrm{R}^{\left.\vec{A}_{r}\right)=\alpha} \vec{B}_{\varphi} \eta\left(\nabla^{2}-R^{-2}\right) \vec{A}_{r}\right.
\end{aligned}
$$

In the right side of the equation (2) include two terms that can produce $B_{\varphi}$ from $B_{r}$, but the first term in the right of equation (3) can produce $B_{r}$ from $B_{\varphi}$, the first term in the right of the equation (2) can be ignored when the rotation is tiny, so $B_{\varphi}$ and $B_{r}$ are produced only by the $\alpha$ effect, the dynamo under this kind of condition is called the ' $\alpha$ '-dynamo. If the rotation speed of the sun plasmas is so great as to ignore the second term in the right of equation (2), $B_{\varphi}$ and $B_{r}$ are maintained by differential rotation and $\alpha$ effect, this kind of the dynamo is called ' $\alpha \omega$ '-dynamo. On this foundation, the people obtain various of model of the dynamo by discuss the differential physics meaning of $\alpha$. 


\section{Results and discussion:}

So there are a lot of articles about the magnetic dynamo henceforth. In 1992, C. A. Tout and J. E. Pringle put forward a simple model of magnetic dynamo by applying the instability of the Parker, the instability of the Balbus-Hawley and the reconnection. While this article also put forward a model of dynamo by applying the above three principles, the difference is that we applying the Sweet-Parker reconnection contrast to the Petschek reconnection, and discuss that whether it can form a self-sustaining magnetic dynamo. Then we can conclude that it can form a self-sustaining magnetic dynamo in this condition, its form of solution is never steady but hovers round about the equilibrium values.

Define $v_{r}=V_{r} / C_{s}, \quad v_{\varphi}=V_{\varphi} / C_{s}, \quad v_{z}=V_{Z} / C_{s}, \quad w_{r}=\frac{v_{r}}{v_{r}{ }^{e q}}, \quad w_{\varphi}=\frac{v_{\varphi}}{v_{\varphi}^{e q}}, \quad w_{z}=\frac{v_{Z}}{v_{Z}{ }^{e q}}$, we can get a complete equation of magnetic dynamo:

$$
\begin{aligned}
& \left\{\begin{array}{l}
\frac{d w_{r}}{d \tau}=a_{1} w_{Z}-a_{2} w_{r} w_{\varphi} \\
\frac{d w_{\varphi}}{d \tau}=a_{3} w_{r}-a_{2} w_{\varphi}{ }^{2} \\
\frac{d w_{Z}}{d \tau}=a_{4} w_{\varphi}{ }^{2}-a_{5} \frac{w_{Z}}{w_{\varphi}}
\end{array}\right. \\
& \left\{\begin{array}{l}
\frac{d w_{r}}{d \tau}=-a_{2} w_{r} w_{\varphi} \\
\frac{d w_{\varphi}}{d \tau}=a_{3} w_{r}-a_{2} w_{\varphi}{ }^{2} \\
\frac{d w_{Z}}{d \tau}=a_{4} w_{\varphi}{ }^{2}-a_{6} \frac{w_{Z}{ }^{2}}{w_{\varphi}}
\end{array}\right. \\
& 0<w_{z}<\frac{1}{\sqrt{3}} \\
& w_{Z}>1 \\
& \begin{cases}\frac{d w_{r}}{d \tau}=a_{1}\left[1-\frac{\left(1-\frac{\pi}{\sqrt{2}} v_{Z}^{e q} w_{Z}\right)^{2}}{(1-\sqrt{3})^{2}}\right]^{1 / 2} w_{Z}-a_{2} w_{r} w_{\varphi} & \\
\frac{d w_{\varphi}}{d \tau}=a_{3} w_{r}-a_{2} w_{\varphi}{ }^{2} & \frac{1}{\sqrt{3}}<w_{Z<1}\end{cases}
\end{aligned}
$$

Although the form of above equation and the equation with Petschek reconnection is consistent, the coefficient of the equation have a very big change, among them:

$$
\begin{aligned}
& a_{1}=8.32 \eta \gamma_{\max } \frac{v_{Z}^{e q}}{v_{r}^{e q}} \\
& a_{2}=\frac{2}{5} v_{\varphi}^{e q} \\
& a_{3}=0.9 \eta \frac{v_{r}^{e q}}{v_{\varphi}^{e q}}
\end{aligned}
$$




$$
\begin{aligned}
& a_{4}=0.16 \frac{\left(v_{\varphi}^{e q}\right)^{2}}{v_{Z}{ }^{e q}} \\
& a_{5}=\frac{30 \Gamma \eta^{2}}{\pi \xi v_{\varphi}^{e q}} \\
& a_{6}=3.535 \eta\left(\frac{6 \Gamma}{\xi \pi}\right)^{1 / 2}
\end{aligned}
$$

We can get the general solution of magnetic dynamo through the above equation, show in figure 1 , among them, the order line represent $w_{r}$, dotted line represent ${ }^{w_{\varphi}}$, solid line represent $w_{z}$. We can see that the trend of the solution of equation with the Sweet-Parker reconnection and Petschek reconnection is basically consistent, all of the solution of equation are a form with limitedly flaps, it is do the flapping of top and bottom around an equilibrium, its period of flapping is united to the period of flapping with Petschek reconnection. The difference is that the average energy with Sweet-Parker reconnection is larger than the average energy with the Petschek reconnection, this because the reconnection time become slowly, the consume of energy is slowly, so the average energy with Sweet-Parker reconnection is larger than the average energy with the Petschek reconnection

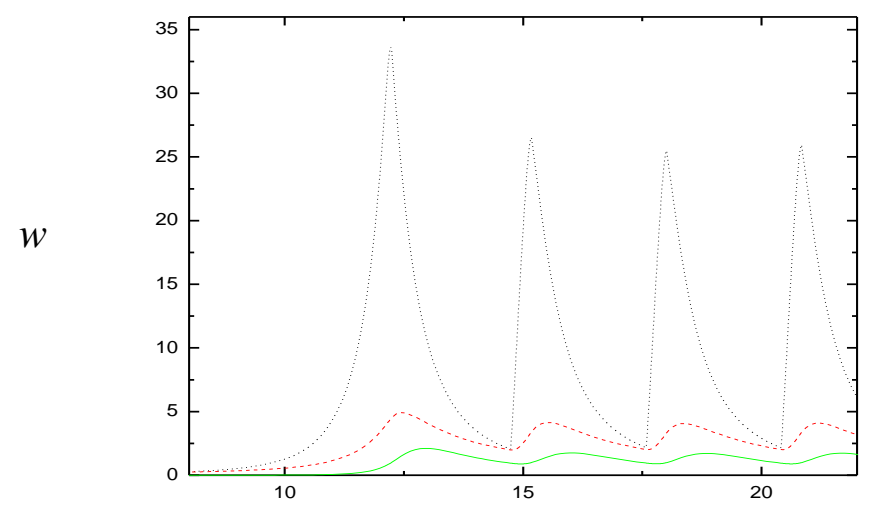

$\tau$

Figure 1.

We can see that the average energy with Sweet-Parker reconnection is larger than the average energy with the Petschek reconnection, its period is 2.8 , similar to the Petschek reconnection. In this situation, the period of flapping is mainly effected by the $w_{Z}$, when the $w_{Z}$ is smaller than 1 , because of the influence of balbus-hawley unsteadying, the $w_{r}$ has a quickly growth, and the $w_{\varphi}$ also has a slowly growth compare to the $w_{r}$, and the growth of $w_{r}$ and $w_{\varphi}$ cause the growth of $w_{Z}$. Contrary to the above situation. when the $w_{Z}$ is bigger than $1, w_{r}$ and $w_{\varphi}$ begin to let up, and the letting up of $w_{r}$ and $w_{\varphi}$ cause the letting up of $w_{Z}$, it has a this kind of circulating. Therefore the form of solutions of equation of magnetic dynamo has a limited form of flapping, it can not indefinitely enlarge as well as indefinitely let up, it will flaps around an equilibrium as the growth of time. 


\section{Conclusions}

We can get a model of self-sustaining magnetic dynamo in accretion disks with Sweet-Parker reconnection through the analysis in this paper, different from the traditional model of magnetic dynamo, this model does not need to have the spiral swift flow athletic field.

\section{Reference}

[1] Larmor J., Brit. Assoc. Reports, 159,1919

[2] Cowling, T. G., Monthly Notices RAS, 94, 39,1934

[3] Elsasser, W. M., Phys. Rev., 69 , 106; 70 , 202.,1946

[4] Parker, E. N., Astrophys. J. 122, 293.,1955

[5] Herzenberg, A., Phil. Trans. Roy. Soc., A249, 507,1957

[6] Krause, F., Radler, K.-H., Mean-Field Magnetohydrodynamics and Dynamo Theory, Pergamon Press, 1980.

[7] Alfvén H., M. N. R. A. S., 107,211,1947

[8] Parker E. N. Ap. J., 160, 383, 1970

[9] Eardley D. M., Lightman A. P., ApJ, 200, 187,1975

[10] Pudritz R., MNRAS, 195, 881,1981a

[11] Pudritz R., MNRAS, 195, 897,1981b

[12] Galeev A. A., Rosner R., Vaiana G. S. ,ApJ, 229, 318,1979

[13] Soward A. M., Astron. Nachr., 299,25,1978

[14] Campbell C. G., Astrophys. Geophys. Fluid Dyn., 63,197,1992

[15] Stepinski T. F., Levy E. H., ApJ, 331, 416,1988

[16] Stepinski T. F., Levy E. H., ApJ, 362, 318,1990

[17] Eardley D. M., Lightman A. P., ApJ, 200,187,1975

[18] C. A. Tout and J. E. Pringle, MNRAS. 259. 604,1992

[19] Frank H. Shu, The Physics of Astrophysics, Vol II, Gas Dynamics. California: University Science Books, 1992

[20] Mouschovias, T. Ch. Astrophys. J. 192, 37,1974

[21] Parker, E. N. Astrophys. J. 145,811,1966

[22] Steven A. Balbus and John F. Hawley, ApJ, 376, 214, 1991

[23]John F. Hawley and Steven A. Balbus, ApJ, 376,223,1991

[24]Evans, C. R., and Hawley, J. F. ApJ, 332, 659,1988

[25] Hawley, J. F., ApJ, 356, 580,1990

[26] ARNAB RAI CHOUDHURI, The Physics of Fluids and Plasmas, Cambridge Univ. Press, Cambridge, 1998

[27] Sweet, P. A. Proc. IAU Symp. 6, 123,1958

[28] Priest, E. R. and Forbes, T. G. J. Geophys. Res. 91, 5579,1986

[29]Furth, H. P., Killeen, J. and Rosenbluth, M. N. Phys. Fluids 6, 459,1963

[30] Dungey, J. W. Phil. Mag. (7) 44, 725,1953

[31] Parker E. N., Cosmical Magnetic Fields. Oxford Univ. Press, Oxford,1979

[32] Wang, X., Ma, Z. W., Bhattacharjee, A. Phys. Plasmas 3(5), 2129, 1996 